\title{
Muon spin relaxation and rotation measurements on single crystals of the heavy-fermion superconductor $\mathrm{UBe}_{13}$
}

\author{
P. Dalmas de Réotier and A. Yaouanc \\ Commissariat à l'Energie Atomique, Département de Recherche Fondamentale sur la Matière Condensée, \\ F-38054 Grenoble Cedex 9, France \\ R. H. Heffner and J. L. Smith \\ Los Alamos National Laboratory, Los Alamos, New Mexico 87545 \\ P. C. M. Gubbens and C. T. Kaiser \\ Interfacultair Reactor Instituut, Delft University of Technology, $2629 \mathrm{JB}$ Delft, The Netherlands
}

(Received 24 February 1999)

\begin{abstract}
We report zero-, longitudinal-, and transverse-field positive muon spin relaxation ( $\mu \mathrm{SR})$ measurements in single crystals of the heavy-fermion superconductor $\mathrm{UBe}_{13}\left[T_{c}=0.83(2) \mathrm{K}\right]$. Our zero-field measurements provide an upper limit for any quasistatic magnetic moments in this system below $100 \mathrm{~K}$ of $0.001 \mu_{B}$, consistent with previous measurements on polycrystals. From the transverse-field measurements we find a lower bound for the London penetration depth $\lambda_{L}=1210 \mathrm{~nm}$. This value implies a lower limit for the effective mass consistent with the low-temperature specific heat.
\end{abstract}

Uranium based heavy-fermion superconductors ( $\mathrm{UPt}_{3}$, $\mathrm{UBe}_{13}, \mathrm{URu}_{2} \mathrm{Si}_{2}, \mathrm{UPd}_{2} \mathrm{Al}_{3}$, and $\mathrm{UNi}_{2} \mathrm{Al}_{3}$ ) have attracted widespread interest because of the strong possibility that the superconducting order parameter in these materials is unconventional, i.e., of lower symmetry than the underlying lattice. Equally intriguing is the possibility that the superconductivity is induced by magnetic interactions between the electrons, rather than by the conventional electron-phonon mechanism. ${ }^{1,2}$ With one exception, all of the uranium-based heavy-fermion superconductors are found to order antiferromagnetically with their Néel temperature larger than their superconducting temperature. The exception is $\mathrm{UBe}_{13}$, which has a cubic crystal structure, a superconducting temperature $T_{c} \simeq 0.85 \mathrm{~K}$, and displays a huge linear coefficient of the specific heat $\left[\gamma=C / T \simeq 1000 \mathrm{~mJ} /\left(\right.\right.$ mole K$\left.\left.^{2}\right)\right] .{ }^{2}$ No magnetic phase transition has been detected in $\mathrm{UBe}_{13}$, despite sensitive muon spin relaxation ${ }^{3-5}$ and neutronscattering experiments. ${ }^{6}$ However, the experimental situation has improved significantly since the earliest $\mu$ SR measurements designed to detect quasistatic magnetic correlations in $\mathrm{UBe}_{13}$. With the advent of the ISIS pulsed muon beam facility (Rutherford Appleton Laboratory, U.K.), one can, in principle, detect magnetic signals much smaller than possible at continuous muon sources. A recent example is presented in Ref. 7, where static magnetic moments as small as $\sim 10^{-4} \mu_{B}$ were detected in the superconductor $\mathrm{CeRu}_{2}$.

In this paper we present zero- and longitudinal-field $\mu \mathrm{SR}$ measurements designed to search for possible magnetic signals of electronic origin in $\mathrm{UBe}_{13}$. In addition, we describe measurements to investigate the superconducting flux-line lattice by the transverse-field $\mu \mathrm{SR}$ method. A priori two sources of magnetic signals are possible: a low-temperature magnetic phase transition or a superconducting phase possessing $\operatorname{spin}^{8}$ or orbital moments. ${ }^{9}$

The $\mu$ SR technique utilizes ${ }^{10-12}$ polarized spin-1/2 muons implanted interstitially into a sample. The muon spin pre- cesses in the local magnetic field until the muon decays (the muon lifetime is $2.2 \mu \mathrm{s}$ ). The temporal evolution of the muon spin is monitored through its decay positron, which is emitted preferentially along the muon spin direction. By collecting several million positrons, one can reconstruct the time dependence of the muon spin depolarization function which, in turn, reflects the spatial and temporal distribution of the magnetic fields at the muon site(s).

The measurements were performed at the MuSR spectrometer of the ISIS pulsed muon beam facility. ${ }^{13}$ Spectra were recorded using a ${ }^{3} \mathrm{He}-{ }^{4} \mathrm{He}$ dilution refrigerator in the temperature range $0.05-4.2 \mathrm{~K}$ and a helium cryostat for temperatures between $1.7 \mathrm{~K}$ and $100 \mathrm{~K}$. The data taken with the two cryostats therefore overlapped in temperature. The sample for this experiment consisted of 1-2-mm-thick $\mathrm{UBe}_{13}$ crystals glued on a silver-backing plate and put together to form a disk of $\sim 32 \mathrm{~mm}$ diam. The [100] crystal direction was perpendicular to the sample plane. In order to ensure that the sample was properly thermalized in the dilution cryostat, we determined the value of $T_{c}$ using zero-fieldcooled transverse-field measurements. ${ }^{14} \mathrm{We}$ found $T_{c}$ $=0.83(2) \mathrm{K}$, consistent with expectation.

We first describe the measurements done with the longitudinal-field geometry from $0.05 \mathrm{~K}$ to $100 \mathrm{~K}$. Here the $\mathrm{Z}$ axis is the direction of the external field and the direction of the initial muon beam polarization. The positrons were detected parallel or antiparallel to the $Z$ direction. In Fig. 1 we present two spectra recorded at $0.4 \mathrm{~K}$, one in zero field and a second in a field of $30 \mathrm{mT}$. The data are well described by the sum of two functions:

$$
P_{Z}(t)=a_{s} P_{Z, s}(t)+a_{b g} \exp \left(-\lambda_{b g} t\right),
$$

where $P_{Z, s}(t)$ describes the relaxation due to the sample and the second term accounts for the muons stopped in the sample holder, cryostat walls and windows. Measurements in 


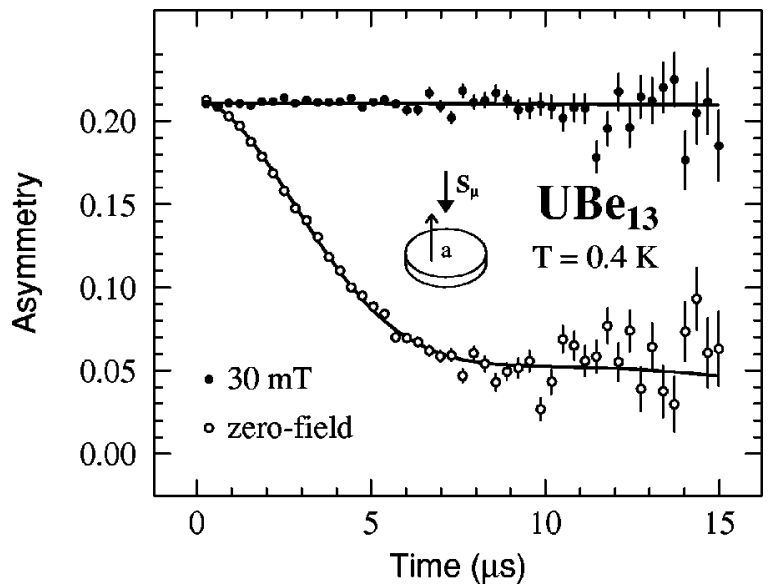

FIG. 1. Typical zero- and longitudinal-field spectra recorded on crystals of $\mathrm{UBe}_{13}$ at $0.4 \mathrm{~K}$. The initial muon beam polarization is parallel to the [100] crystal axis. The lines are the results of fits explained in the main text.

zero field with only the silver plate and no sample show that a good estimate of $\lambda_{b g}$ for the experiments with the dilution refrigerator in zero field is $0.033 \mathrm{MHz}$, for the size of the sample used. For the helium cryostat test measurements have determined that we can take $\lambda_{b g}=0$. The zero-field spectra were fitted with $a_{s}$ and $a_{b g}$ as free parameters, which were found to be constant in temperature, but with different values for the dilution and helium cryostats. In zero field $P_{Z, s}(t)$ was taken to be the Kubo-Toyabe function, ${ }^{15}$ while in longitudinal field an exponential $\exp \left(-\lambda_{Z} t\right)$ was used. In zero field the depolarization due to the sample can therefore be described by:

$$
P_{\mathrm{KT}}(t)=\frac{1}{3} \exp \left(-\frac{2}{3} \nu_{\mu} t\right)+\frac{2}{3}\left(1-\Delta_{Z}^{2} t^{2}\right) \exp \left(-\frac{\Delta_{Z}^{2} t^{2}}{2}\right),
$$

where the Kubo-Toyabe damping rate $\Delta_{Z}=\gamma_{\mu} \sqrt{\left\langle B^{2}\right\rangle_{\mathrm{ZF}}}$ describes the width of the distribution of local fields, $\nu_{\mu}$ is the local-field fluctuation rate as seen by the muon, $\gamma_{\mu}$ is the muon gyromagnetic ratio $\left(\gamma_{\mu}=851.6 \mathrm{Mrad} \mathrm{s}^{-1} \mathrm{~T}^{-1}\right)$, and $\left\langle B^{2}\right\rangle_{\mathrm{ZF}}$ the second moment of the field distribution at the muon site in zero field. An obvious origin from this field distribution stems from the magnetic moments of the ${ }^{9} \mathrm{Be}$ nuclei. We find that $\nu_{\mu}$ is small $[\approx 0.15(5) \mathrm{MHz}]$ and temperature independent within the uncertainties, for the temperature range investigated. Therefore, for $t<5 \mu$ s the depolarization is given, with a good accuracy, by the static KuboToyabe function. The fact that the depolarization due to the sample in zero field is well described by the strong collision Kubo-Toyabe function with a small $\nu_{\mu}$ value is a strong indication that the spins of the muons are depolarized by a quasistatic field distribution. ${ }^{10-12}$ This interpretation was confirmed by longitudinal-field measurements (Fig. 1) that showed negligible depolarization, as expected.

In Fig. 2 we present the temperature dependence of $\Delta_{Z}$ and $\lambda_{Z} \cdot \lambda_{Z}$ is essentially zero and $\Delta_{Z}$ is temperature independent with a value of 0.252 (8) $\mathrm{MHz}$. The width of the field distribution, $\Delta_{Z, n} / \gamma_{\mu}$, at the muon site arising from the ${ }^{9} \mathrm{Be}$ nuclear magnetic moment can be computed if the muon site is known. This site has been determined from other high

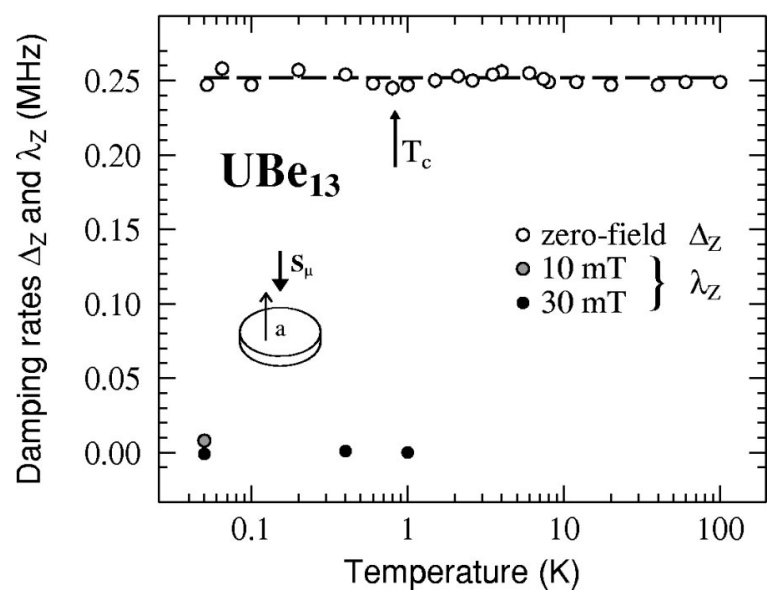

FIG. 2. Temperature dependence of the Kubo-Toyabe damping rate $\Delta_{Z}$ and the exponential damping rate $\lambda_{Z}$ measured on crystals of $\mathrm{UBe}_{13}$. The initial muon beam polarization is parallel to the [100] crystalline axis. The dashed straight line indicates the average $\Delta_{Z}$ value. Since $\Delta_{Z}$ is temperature independent, we infer an upper limit for any quasistatic magnetic correlations of $0.001 \mu_{B}$ in $\mathrm{UBe}_{13}$. In addition, below the superconducting transition $\left[T_{c}\right.$ $=0.83(2) \mathrm{K}$, as determined in this experiment], we do not detect any magnetic field due to the Cooper pairs. The quenching of the zero-field damping by a small applied longitudinal field confirms that the field distribution is quasistatic, and is due to the ${ }^{9} \mathrm{Be}$ nuclear moments.

transverse-field measurements ${ }^{16}$ to be at the $(0,1 / 4,1 / 4)$ site ( $24 d$ in Wyckoff notation, space group $F m 3 c$ ), i.e., halfway between two uranium ions. The computation of $\Delta_{Z, n} / \gamma_{\mu}$ is performed in a two-step process. First, taking into account the nuclear dipolar fields and neglecting the electric-field gradients (EFG) acting on the ${ }^{9} \mathrm{Be}$ nuclei we compute $\Delta_{Z, n}$ $=0.277 \mathrm{MHz} .{ }^{17}$ Secondly, the effect of the large EFG present in $\mathrm{UBe}_{13}$ (Ref. 3) is accounted for by decreasing $\Delta_{Z, n}$ by $\simeq 10 \%$. ${ }^{18}$ Therefore, the computed and experimental values are in agreement. If there were an extra static-field distribution of electronic origin characterized by a width $\Delta_{Z, e} / \gamma_{\mu}$, we would have $\Delta_{Z}^{2}=\Delta_{Z, n}^{2}+\Delta_{Z, e}^{2}$. From the uncertainty in $\Delta_{Z}(\delta=0.008 \mathrm{MHz})$ we deduce an upper bound on $\Delta_{Z, e}: \Delta_{Z, e} \leqslant 0.09 \mathrm{MHz} .{ }^{19}$ Therefore, any change in the internal quasistatic magnetic field induced by magnetism or superconductivity has to be smaller than $\Delta_{Z, e} / \gamma_{\mu}$ $=0.11 \mathrm{mT}$ at the muon site over the whole temperature range investigated $(0.05-100 \mathrm{~K})$. We note that our measured $\Delta_{Z}$ value is consistent with results obtained previously in polycrystalline samples. ${ }^{4}$ With a model similar to the one used for estimating $\Delta_{Z, n} / \gamma_{\mu}$, we can compute the field distribution width $\Delta_{Z, e} / \gamma_{\mu}$ at the muon site originating from spatially disordered quasistatic electronic uranium moments. We find that uranium moments of $10^{-3} \mu_{B}$ would produce a field width of $0.11 \mathrm{mT}$. Therefore, we conclude that if the uranium ions carry a magnetic moment, it is very small: $\mu_{\mathrm{U}} \lesssim 10^{-3} \mu_{B}$.

We stress that, while our results strongly suggest that there are no (or very small) quasistatic magnetic correlations in $\mathrm{UBe}_{13}$, we do not exclude the possibility of short-lifetime magnetic fluctuations. In fact, such fluctuations have already been observed on a powder sample by inelastic neutron scattering. ${ }^{6}$ 
An alternative interpretation of the data consists of examining the case of a magnetic order, with an appreciable correlation length, of the uranium ions. In such a system, one would generally observe a spontaneous muon precession, the frequency of which depends on the magnitude of the uranium moments. If this frequency is small enough, the depolarization function has a parabolic shape within the $\mu \mathrm{SR}$ time window. The observed muon depolarization function would then be the product of this parabolic function by the KuboToyabe function stemming from the ${ }^{9} \mathrm{Be}$ nuclear moments. With the same procedure as in the case of the spatially disordered electronic moments, the upper bound of the $t^{2}$ coeffcient of the parabola would be $0.09 \mathrm{MHz}$ to the square, corresponding to a field at the muon site of $0.15 \mathrm{mT}$. Although the precise value of the uranium moment that would yield such a field at the muon site depends on the type of the magnetic ordering, we find again that values of the ordered moment of $\sim 10^{-3} \mu_{B}$ would produce this field magnitude.

We now discuss the implications of these measurements, noting first that we do not detect any signature of quasistatic magnetic correlations down to $0.05 \mathrm{~K}$. Knowing the extreme sensitivity of the zero-field $\mu \mathrm{SR}$ technique to these correlations, ${ }^{11,12}$ our results suggest that $\mathrm{UBe}_{13}$ does not have a magnetic phase transition, and is therefore the only uranium heavy-fermion superconductor with no magnetic phase transition. This conclusion is weakened somewhat by the fact that the $\mu \mathrm{SR}$ technique does not detect the magnetic phase transition at $5 \mathrm{~K}$ in good quality samples of $\mathrm{UPt}_{3}$, even though this transition was seen by neutron and x-ray magnetic diffraction. ${ }^{20}$ Therefore, there is still a possibility that $\mathrm{UBe}_{13}$ has a phase transition that can not be observed by zero field $\mu \mathrm{SR}$ because either (1) the sum of the quasistatic dipolar fields from the uranium moments at the muon site is zero for the magnetic structure adopted by the compound or (2) the uranium moments fluctuate faster than $10^{-6}-10^{-7} \mathrm{~s}$. These two possibilities have been proposed to explain the nonobservation by zero field $\mu$ SR of the $5-\mathrm{K}$ magnetic phase transition in some samples of $\mathrm{UPt}_{3}$.

Within the experimental uncertainty, $\Delta_{Z}$ has the same value above and below the superconducting temperature. Thus we do not observe any magnetic moment due to a possible time-reversal violating order parameter in the superconducting phase. Moments due to such a phase can have two possible origins: the undamped ring currents that produce an orbital field or, if the Cooper pairs have a spin, an additional field due to the spin density. The former field is expected to be much smaller than the latter. Mineev has estimated that the field due to the spin density should be $\sim 0.1$ $\mathrm{mT}$ if the Cooper pair is in a triplet state. ${ }^{8}$ Since this theoretical value is close to the upper bound given by our measurements, we cannot draw a definite conclusion regarding the state of the Cooper pair electrons in $\mathrm{UBe}_{13}$.

The transverse $\mu \mathrm{SR}$ technique can be a very efficient technique to investigate the physical properties of the fluxline lattice in the superconducting state if the magnetic penetration depth $\lambda$ is small enough, i.e., if the field distribution due to the vortices is sufficiently large. ${ }^{11}$ In an attempt to study this vortex-induced field distribution we recorded six spectra above $T_{c}$ (typically at $1 \mathrm{~K}$ and $2 \mathrm{~K}$ ) and six spectra far below $T_{c}$ (at $0.05 \mathrm{~K}$, with one measurement at $0.1 \mathrm{~K}$ ). The measurements were done in an applied magnetic field
$B_{\text {ext }}$ of $5 \mathrm{mT}, 20 \mathrm{mT}$, and $35 \mathrm{mT}$. In this geometry, the direction of the initial muon beam polarization and the positron detectors are denoted as $X$, perpendicular to $Z$. All the spectra are properly described by the sum of two functions:

$$
P_{X}(t)=a_{s} \exp \left(-\frac{\Delta_{X}^{2} t^{2}}{2}\right) \cos \left(\omega_{s} t\right)+a_{b g} \cos \left(\omega_{b g} t\right),
$$

where the first term describes the signal due to the sample and the second accounts for the muons stopped in the sample environment. Here $\omega_{i}=\gamma_{\mu} B_{i}$, where $B_{i}$ is the mean value of the magnetic field probed by the muon in the sample $(i$ $\equiv s)$ and the sample environment $(i \equiv b g)$. We found that $\omega_{s} \simeq \omega_{b g}$. Within the experimental uncertainty, $\Delta_{X}$ was found to have the same value above and below $T_{c}$. We measured $\Delta_{X}=0.256$ (5) $\mathrm{MHz}, 0.236$ (5) $\mathrm{MHz}$, and 0.220 (7) $\mathrm{MHz}$ for $B_{\text {ext }}=5,20$, and $35 \mathrm{mT}$, respectively. Thus $\Delta_{X}$ decreases slightly with applied magnetic field.

Again, as in the zero-field case, we expect that the ${ }^{9} \mathrm{Be}$ nuclear moments produce a field distribution at the muon site that results in the Gaussian signal observed in the experiments. The presence of vortices produce an additional source of damping of the muon depolarization. Using the methodology introduced for the analysis of the zero-field data, we can estimate an upper bound for the width of the field distribution due to the flux-line lattice $\Delta_{X, v}$. From the data recorded at $B_{\text {ext }}=5 \mathrm{mT}$ and $20 \mathrm{mT}$ (the more precise measurements) we find $\Delta_{X, v} \leqslant 0.07 \mathrm{MHz}$. Using the relation ${ }^{21}$

$$
\frac{\Delta_{X, v}}{\gamma_{\mu}}=0.06092 \frac{\Phi_{0}}{\lambda^{2}},
$$

where $\Phi_{0}=2.07 \times 10^{-15} \mathrm{Tm}^{2}$ is the quantum of flux, we deduce that $\lambda \geqslant 1240 \mathrm{~nm}$. Since the ratio $B_{\text {ext }} / B_{c 2}$ is extremely small [the upper critical field $B_{c 2} \approx 9 \mathrm{~T}$, (Ref. 22)], we have neglected the effect of the vortex cores in Eq. (4). ${ }^{23}$

The measured upper bound of the penetration depth is, in fact, an effective value since it is influenced by the impurities contained in the sample. The London penetration depth at $T=0, \lambda_{L}$, can be obtained using $\lambda_{L}=\lambda(1+\xi / l)^{-1 / 2}$, where $\xi$ is the coherence length and $l$ the mean free path. ${ }^{24}$ We take $\xi=10 \mathrm{~nm}$ (Ref. 22) and estimate $l$ from the residual resistivity measured at $T=0$ and in high-magnetic field since the zero-field resistivity is strongly influenced by the spin fluctuations. ${ }^{2}$ From the free-electron model $^{25}$ and a value of the residual resistivity of $15 \mu \Omega \mathrm{cm}$, we derive $l=180 \mathrm{~nm}$, i.e., our $\mathrm{UBe}_{13}$ crystals are in the clean limit $(l \gg \xi)$. Thus we get $\lambda_{L} \geqslant 1210 \mathrm{~nm}$.

We now derive bounds on the lower critical field and effective mass from the $\lambda_{L}$ value. We have $B_{c 1}$ $=\Phi_{0} \ln (\kappa) /\left(4 \pi \lambda_{L}^{2}\right)<0.54 \mathrm{mT}$. The effective mass $m^{*}$ is expressed in terms of $\lambda_{L}$ and the superfluid density $n_{s}$ as $m^{*}$ $=\lambda_{L}^{2} \mu_{0} n_{s} e^{2}$ (Ref. 24) [or $m^{*}=\lambda_{L}^{2}\left(4 \pi n_{s} e^{2}\right) / c^{2}$ in cgs units]. Hall measurements yield an average value $n_{s}$ $=3.2 \times 10^{20} \mathrm{~cm}^{-3} \cdot{ }^{26,27}$ Thus we obtain $m^{*}>16.5 m_{e}$, where $m_{e}$ is the free-electron mass. This lower bound is consistent with the effective mass derived from the low-temperature Sommerfeld constant, which is $m^{*} \approx 250 m_{e} \cdot{ }^{2}$

Finally, we briefly discuss the magnitude of the transverse-field linewidth. We find no evidence for internal 
fields of an electronic origin in either the longitudinal or transverse geometries, and find no change in linewidth with temperatures above and below $T_{c}$. Therefore, the measured linewidths $\Delta_{X}$ must only be due to the ${ }^{9}$ Be nuclear moments. We note that $\Delta_{X} \simeq \Delta_{Z}$. As mentioned in Ref. 3, this is explained by the effect of the large EFG. The slight decrease of $\Delta_{X}$ when the applied field is increased reflects the competition between the electric quadrupolar and Zeeman energies on the ${ }^{9} \mathrm{Be}$ nuclei. In the limit of high-magnetic field, we compute $\Delta_{X}=0.19 \mathrm{MHz}$. This is not far from the measured value at our largest field: $\Delta_{X}=0.220$ (7) $\mathrm{MHz}$.

In conclusion, our zero-field $\mu$ SR measurements on single crystals of $\mathrm{UBe}_{13}$ provide an upper limit for any quasistatic moments in this system below $100 \mathrm{~K}$ of $0.001 \mu_{B}$. This is in agreement with previous measurements on polycrystals. ${ }^{4}$ In addition, we measured a lower bound for the London penetration depth $\lambda_{L}>1210 \mathrm{~nm}$. This yields a lower bound for the effective mass $m^{*}>16.5 m_{e}$, which is consistent with the effective mass derived from the low-temperature specific heat.

The researchers from the Netherlands acknowledge support from the Dutch Scientific Organization (NWO). The work at Los Alamos was carried out under the auspices of the U.S. D.O.E.
${ }^{1}$ N. Grewe and F. Steglich, in Handbook on the Physics and Chemistry of Rare Earths, edited by Karl A. Gschneider, Jr. and LeRoy Eyring (North Holland, Amsterdam, 1991), Vol. 14.

${ }^{2}$ R.H. Heffner and M.R. Norman, Comments Condens. Matter Phys. 17, 361 (1996).

${ }^{3}$ R.H. Heffner, D.W. Cook, A.L. Giorgi, R.L. Hutson, M.E. Schillaci, D.E. MacLaughlin, C. Boekema, R.L. Lichti, J. Oostens, and A.B. Denison, Phys. Rev. B 39, 11345 (1989).

${ }^{4}$ R.H. Heffner, J.L. Smith, J.O. Willis, P. Birrer, C. Baines, F.N. Gygax, B. Hitti, E. Lippelt, H.R. Ott, A. Schenck, E.A. Knetsch, J.A. Mydosh, and D.E. MacLaughlin, Phys. Rev. Lett. 65, 2816 (1990).

${ }^{5}$ G.M. Luke, L.P. Le, B.J. Sternlieb, W. D. Wu, Y.J. Uemura, J.H. Brewer, R. Kadono, R.F. Keifl, S. R. Kreirzman, T.M. Riseman, Y. Dalichaouch, B.W. Lee, M.B. Maple, C.L. Seaman, P.E. Armstrong, R.W. Ellis, Z. Fisk, and J.L. Smith, Phys. Lett. A 157, 173 (1991).

${ }^{6}$ G.H. Lander, S.M. Shapiro, C. Vettier, and A.J. Dianoux, Phys. Rev. B 46, 5387 (1992).

${ }^{7}$ A.D. Huxley, P. Dalmas de Réotier, A. Yaouanc, D. Caplan, M. Couach, P. Lejay, P.C.M. Gubbens, and A.M. Mulders, Phys. Rev. B 54, R9666 (1996).

${ }^{8}$ V.P. Mineev, Zh. Éksp. Teor. Fiz. 49, 624 (1989) [JETP Lett. 49, 719 (1989)].

${ }^{9}$ C.H. Choi and P. Muzikar, Phys. Rev. B 39, 9664 (1989).

${ }^{10}$ A. Schenck and F.N. Gygax, in Handbook of Magnetic Materials, edited by K.H.J. Buschow (Elsevier Science, New York, 1995), Vol. 9.

${ }^{11}$ P. Dalmas de Réotier and A. Yaouanc, J. Phys.: Condens. Matter 9, 9113 (1997).

${ }^{12}$ A. Amato, Rev. Mod. Phys. 69, 1119 (1997).

${ }^{13}$ G.H. Eaton, C.A. Scott, and W.G. Williams, Hyperfine Interact.
85, 1099 (1994).

${ }^{14}$ G.M. Luke, A. Keren, L.P. Le, W.D. Wu, Y.J. Uemura, D.A. Bonn, L. Taillefer, and J.D. Garrett, Phys. Rev. Lett. 71, 1466 (1993).

${ }^{15} \mathrm{R}$. Kubo and T. Toyabe, in Magnetic Resonance and Relaxation (North-Holland, Amsterdam, 1967), p. 810.

${ }^{16}$ R.H. Heffner et al. (unpublished).

${ }^{17}$ R.S. Hayano, Y.J. Uemura, J. Imazato, N. Nishida, T. Yamazaki, and R. Kubo, Phys. Rev. B 20, 850 (1979).

${ }^{18}$ P. Dalmas de Réotier, Ph.D. thesis, INPG, Grenoble, 1990.

${ }^{19}$ Let us suppose that the field distribution at the muon site has two origins. If each of the two distributions is Gaussian, the widths add in quadrature: $\Delta^{2}=\Delta_{1}^{2}+\Delta_{2}^{2}$. Supposing that $\Delta_{1} / \Delta_{2} \ll 1$, we get $\Delta_{1} \simeq 2 \sqrt{\delta \Delta_{2}}$ with $2 \delta \equiv \Delta-\Delta_{2}$.

${ }^{20}$ P. Dalmas de Réotier, A. Huxley, A. Yaouanc, J. Flouquet, P. Bonville, P. Imbert, P. Pari, P.C.M. Gubbens, and A.M. Mulders, Phys. Lett. A 205, 239 (1995).

${ }^{21}$ E.H. Brandt, Phys. Rev. B 37, 2349 (1988).

${ }^{22}$ M.B. Maple, J.W. Chen, S.E. Lambert, Z. Fisk, J.L. Smith, H.R. Ott, J.S. Brooks, and M.J. Naughton, Phys. Rev. Lett. 54, 477 (1985).

${ }^{23}$ A. Yaouanc, P. Dalmas de Réotier, and E.H. Brandt, Phys. Rev. B 55, 11107 (1997).

${ }^{24}$ Michael Tinkham, Introduction to Superconductivity (McGrawHill, New York, 1996).

${ }^{25}$ Neil W. Ashcroft and N. David Mermin, Solid State Physics (Holt, Rinehart, and Winston, New York, 1976), p. 757.

${ }^{26}$ T. Penney, J. Stankiewicz, S. von Molnar, Z. Fisk, J.L. Smith, and H.R. Ott, J. Magn. Magn. Mater. 54-57, 370 (1986).

${ }^{27}$ N.E. Alekseevskii, V.N. Narozhnyi, V.I. Nizhankovskii, E.G. Nikolaev, and E.P. Khlybov, Zh. Eksp. Teor. Fiz. 40, 421 (1984) [JETP Lett. 40, 1241 (1984)]. 